\title{
Contents of Volume I
}

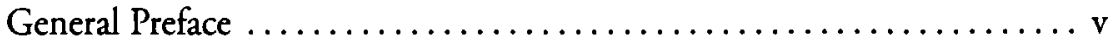

Preface to Volume I .................................. ix

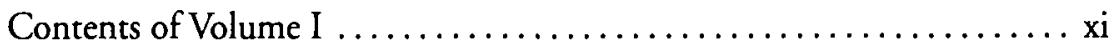

Contents of Volume II .............................. xvi

Contents of Volume III $\ldots \ldots \ldots \ldots \ldots \ldots \ldots \ldots \ldots \ldots \ldots \ldots \ldots \ldots \ldots$

List of Contributors ............................ xxvii

Opening Addresses

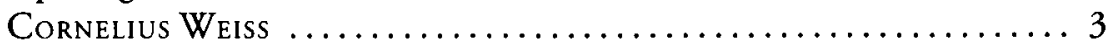

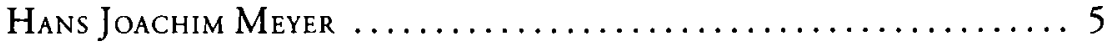

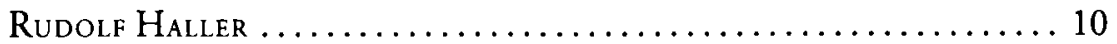

Opening Lecture

DagfinN Føllesdal

Was ist Analytische Philosophie? ........................ 15

I. Logic

BERND W. BuLdT

Anmerkungen zum Beweis des zweiten Gödelschen

Unvollständigkeitssatzes ............................ 31

Michael Dummett

Existence, Possibility and Time $\ldots \ldots \ldots \ldots \ldots \ldots \ldots \ldots \ldots . \ldots . \ldots . \ldots$

André FuhrmanN

Everything in Flux: Dynamic Ontologies ................. 68

Siegrried Gottwald

Fuzzy-Logik und approximatives Schließen - ein kurzer Überblick .... 78

Volker Halbach

Classical Symmetric Truth ............................ 87 
Ho Ngoc Duc

On a Dilemma of Conditional Obligation

Bertram Kienzle

„Es wird der Fall sein, daß"

Karl-Heinz Krampitz

Negation und negative Existenzaussagen

Herbert Kústner

Dreiwertige Logik und dynamische Semantik

Uwe Meixner

Ontologically Minimal Semantics for Intuitionistic Logic ..

Ulrich Metschl

What is a Logical Constant? A Topological Suggestion

Hans RotT

Vom Primat der praktischen Vernunft. Logische Regeln als Regeln rationaler Wahl

Mark Siebel

Bolzanos Ableitbarkeit und Tarskis Logische Folgerung ...

Hermann Weidemann

Eine durch Abstraktion gewonnene Definition der Zeit ..

Horst Wessel

Wider den Mythos intensionaler Kontexte

Klaus Wuttich

Intensional genannte Kontexte

ERnst Zimmermann

Rationalitätsgrade in schwachen Glaubenslogiken . .

II. Epistemology

Thomas Bartelborth

Ist der frühe Carnap ein geeigneter Gegenspieler für Quine?

Elke Brendel

Was können wir über das Wissen wissen? Erkenntnistheoretische Betrachtungen aus semantischer Perspektive .. 
Wolfgang Deppert

Hierarchische und ganzheitliche Begriffssysteme

Bernward Gesang

Austin contra Frege. Ein Versuch zur Klärung der

Korrespondenztheorie

Thomas Grundmann

Die Bedingungen des Verstehens als Bedingungen der Gegenstände des Verstehens

Reinhard Kleinknecht

Wissen, Glauben und Wünschen

Kurt Koleznik

Meinungszirkel, epistemisches Prinzip und Supervenienz

Verena Mayer

Logischer Holismus: Zur Erkenntnistheorie des Tractatus

N. M. L. Nathan

Stoics and Sceptics

Alexander Roesler

Kritik der Searleschen Wahrnehmungstheorie

WOLFGANG SPOHN

Über die Gegenstände des Glaubens

Renate Wahsner

Über die Berechtigung des analytischen Vorgehens

in der Philosophie

Nicholas White

Widerlegt sich der protagoreische Relativismus selbt?

III. Philosophy of Science

WOLFGANG BALZER

Referenz in wissenschaftlichen Theorien

Andreas Bartels

Bedeutungsketten. Ein Modell der Begriffsbildung in gegenwärtigen physikalischen Theorien 
xiv

ThOMAs Bonk

Vollständige Theorien

ULRICH GäHDE

Theorien-Netze und konsistente Modellbildung

Michaela HaAse

Der Begriff Pragmatischer Idealisierung

Stephan Hartmann

Verfolgt die Elementarteilchenphysik ein reduktionistisches Programm? 374

Reiner Hedrich

Fand eine „Revolution des Komplexen“ statt? ............... 381

Kay Herrmann

Zur Raum-Zeit Konzeption der modernen Physik

Paul Hoyningen-Huene

Die neuzeitliche Naturerkenntnis zerstört die Natur. Zu Georg Pichts

Theorie der modernen Naturwissenschaften

Max Kistler

Zur Transfer-Theorie der Kausalität

Bernhard Lauth

c-Komplexität und exakte Identifikation

Agnieszka Lekka-Kowalik

Popper, Kuhn and Laudan on the Rationality of Science:

A Shared View

Thomas Mormann

Repräsentation, Struktur, Quasianalyse. Formale Aspekte einer

Carnapianischen Konstitutionstheorie

IlkKa Niniluoto

Theoretical Reference and Truthlikeness

Arne Schirrmacher

Submikroskopische Vorstellungen von Raum und Zeit - eine

Herausforderung für die (analytische) Philosophie?

Christina Schneider

Häufigkeitsauffassung der Wahrscheinlichkeit - insignifikant ohne ein "metaphysisches Konzept" 
Daniel Schoch

Ein topologisches Einfachheitskriterium zur rationalen

Theorienwahl

Gerhard Schurz

Unification and Understanding ...

Gerhard Terton

Beispiele in der methodologischen Erklärungsdiskussion

Max URCHS

Der Bruch der starken Kausalität 502

\section{Appendix}

Georg Meggle

Erweiterter Bericht des Präsidenten vor der

2. Mitgliederversammlung der Gesellschaft für Analytische

Philosophie (GAP) am 9.9.1994 in Leipzig

\section{Indices}

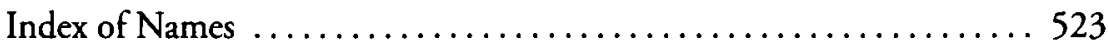

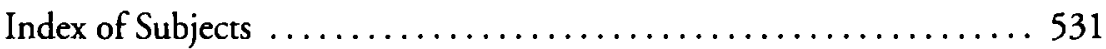

